\title{
A versatilidade do K-10, como suporte sólido, em reações orgânicas
}

\author{
The versatility of K-10 as solid support in organic reactions \\ Hugo T. Schmitz Braibante', Mara E. Fortes Braibante ${ }^{2}$ \\ 1,2 Universidade Federal de Santa Maria, Santa Maria, Brasil.
}

\begin{abstract}
Resumo
Montmorilonita, K-10 é amplamente utilizada em reações orgânica, pertence à classe dos K-catalisadores, derivados de um mineral de hidrossilicato de alumínio. O K-10 apresenta uma estrutura em forma de "sanduíche", na qual uma camada octaédrica (Al) é intercalada entre duas tetraédricas (Si). A Montmorilonita, K10, vem sendo utilizada como catalisador em reações orgânicas em processos nos quais são utilizados como catalisadores ácidos de Lewis ou de Brönsted. Outra aplicação é seu uso como suporte sólido ancorando reagentes, como os oxidantes Clayfen e Claycop, impregnado de metais como Fe3+, Zn2+, Co 2+, usados como catalisador em reações de condensação, em MCR's e atualmente com prolina como catalisador bifuncional. Cabe destacar a possibilidade de usar as reações em K10 associadas a várias formas de energia, refluxo em solventes orgânicos, em micro-ondas ou ultrassom e atendendo a preceitos da química verde em reações sem solventes. Várias rotas sintéticas e interconversão de grupos funcionais são enfocadas como transesterificação, halogenação, oxidação aliadas as clássicas reações de Acilação e alquilação de aromáticos, reação de Diels Alder e rearranjos que levam sistemas heterocíclicos como Pirazóis, Isoxazóis, Isoquinolinas, Pirazolonas e Isoxazolonas. Todas estas propriedades e aplicações ressaltama versatilidade do K-10, como suporte sólido, em reações orgânicas.
\end{abstract}

Palavras-chave: Montmorilonita, suporte sólido, K10, heterociclos, enamino compostos.

\begin{abstract}
Montmorillonite $\mathrm{K}$ - 10 is widely used in organic reactions, belongs to the class $\mathrm{K}$-catalysts derived from a mineral aluminum hydrosilicate. K- 10 has a structure in the form of "sandwich "in which an octahedral layer $(A l)$ is sandwiched between two tetrahedral (Si). The Montmorillonite, K10, has used as catalyst in organic reactions in processes where acids are used as catalysts Lewis or Brönsted. Another application is their use as solid support anchoring reagents such as Clayfen and Claycop oxidants, impregnated with metals such as Fe3 +, Zn 2 +, Co $2+$, used as a catalyst in condensation reactions in MCR 's and currently proline as bifunctional catalyst. It is worth noting the possibility of using the K10 reactions associated with various forms of energy, reflux in organic solvents in microwave or ultrasound and given the precepts of green chemistry in reactions without solvents. Several synthetic routes and interconversion of functional groups are focused as transesterification, halogenation, oxidation combined with the classic reactions of aromatic acylation and alkylation, Diels Alder and rearrangements leading heterocyclic systems such as Pyrazoles, Isoxazoles, Isoquinolines, Pyrazolones and Isoxazolonas. All these properties and applications highlight the versatility of the K-10 as solid support in organic reactions.
\end{abstract}

Keywords: Montmorillonite, solid support, K10, heterocyclic compounds, enamino compounds. 


\section{Introdução}

$\mathrm{O}$ $s$ químicos vem utilizando reagentes imobilizados em sólidos finamente divididos desde adécada de 20, que foram chamados de suportes sólidos por Posnerem 1978. Os suportes sólidos podem ser divididos em dois grupos, segundo a sua natureza: polímeros orgânicos, nos quais os reagentes são ligados covalentemente ao suporte conhecidas como Resina de Merrifield, EPZG (MERRIFIELD, 1963) e os sólidos inorgânicos, nos quais os reagentes são adsorvidos, dentre estes suportes podemos destacar os argilo minerais.

\section{Argilo Minerais}

Os argilo minerais são sólidos inorgânicos os quais vêm sendo muito empregados na síntese orgânica nos últimos anos. As estruturas atômicas dos argilo minerais comuns têm sido determinadas por inúmeros investigadores, que se basearam em estudos generalizados feitos por Pauling, sobre a estrutura das micas e minerais relacionados (PAULING, 1930).

Os argilo minerais geralmente apresentam duas unidades estruturais básicas: uma estrutura primária e uma estrutura secundária. A estrutura primária consiste em duas folhas formadas por oxigênios e/ou hidroxilas, com as quais um cátion metálico (alumínio, ferro ou magnésio) está coordenado octaedricamente. $O$ cátion fica equidistante dos seis oxigênios e/ou hidroxilas (Figura 1). Já a estrutura secundária é constituída de tetraedros de silício. Em cada tetraedro, o átomo é equidistante dos quatro oxigênios ou hidroxilas (Figura 2).

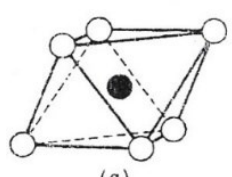

(a)

O e/ou $:$ i $=$ hidroxilas

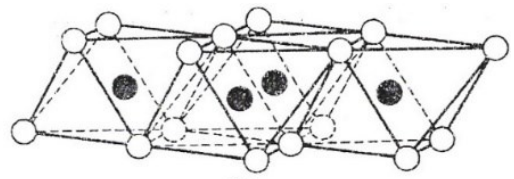

(b)

aluminio, magnésio, etco
Figura 1 - Estrutura primária que faz parte da constituição de um argilo mineral.

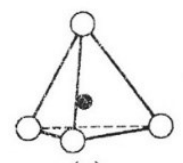

(a)

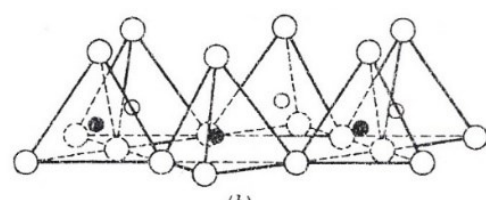

(b)
$O \cdot O=$ Oxigënios $\quad O=0$ Silicios

Figura 2 - Estrutura secundária que faz parte da constituição de um argilo mineral.

Os argilo minerais são classificados de acordo com o número relativo de folhas tetraédricas e octaédricas que constituem suas camadas. Os três principais arranjos encontrados são formados por:

a) camadas constituídas por uma folha tetraédrica alternada com uma folha octaédrica (estrutura conhecida como (1:1)). Ex.: caulinitas;

b) camadas constituídas na forma de sanduíche nas quais uma folha octaédrica encontra-se entre duas folhas tetraédricas (estrutura conhecida como (2:1)). Ex.: montmorilonita;

c) camadas constituídas por estruturas (2:1) alternadas com estruturas (2:1:1). Ex.: clorita.

\subsection{Montmorilonita (K-10)}

Uma das argilas mais utilizadas em reações na química orgânica é a montmorilonita, K-10, que pertence à classe dos $\mathrm{K}$-catalisadores, derivados de um mineral de hidrossilicato de alumínio. O K-10 apresenta uma estrutura em forma de "sanduíche", na qual uma camada octaédrica é intercalada entre duas tetraédricas. Os tetraedros de silício da estrutura secundária estão ligados uns aos outros por meio de três vértices, formando uma rede hexagonal (Figura 3). O quarto vértice de cada tetraedro está associado a um vértice de um octaedro de alumínio da estrutura primária, formando uma camada em comum (Figura 3).

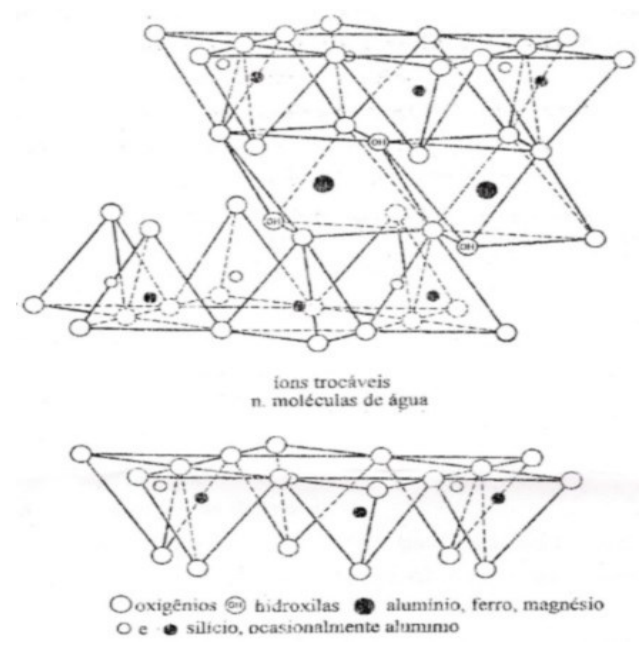

Figura 3 - Estrutura lamelar da montmorilonita, K-10.

Devido à unidade empilhada de sílica-alumina-sílica, os oxigênios de cada tetraedro ou octaedro são adjacentes aos oxigênios da camada vizinha, e como consequência, têm uma fraca ligação e uma excelente clivagem entre as camadas.

Uma das mais interessantes características da estrutura montmorilonita é a de que água e outras moléculas polares, como certas moléculas orgânicas, podem penetrar entre as camadas, causando sua expansão. Os cátions trocáveis localizam-se entre essas camadas e o tamanho do espaço interlamelar da montmorilonita, completamente desidratada, depende somente do tamanho dos cátions interlamelares. Os principais cátions presentes na 
montmorilonita são $\mathrm{Na}^{+}, \mathrm{K}^{+}, \mathrm{H}_{3} \mathrm{O}^{+}, \mathrm{Mg}^{2+}, \mathrm{Ca}^{2+}, \mathrm{Al}^{3+} \mathrm{e} \mathrm{Fe}^{3+}$.

A propriedade de expansão é reversível, a não ser que a estrutura seja completamente colapsada pela remoção de todas as moléculas polares interlamelares que podem dificultar ou impossibilitar a expansão da estrutura.

Outra característica relevante da montmorilonita, K-10 refere-se a sua grande área superficial (500-760 $\mathrm{m}^{2} / \mathrm{g}$ ), que é decorrente da sua constituição por partículas muito finas com dimensões médias de $2 \mu$.

Outra propriedade do K-10 é a sua acidez superficial. Essa acidez é decorrente dos grupos hidroxilas terminais e da interação entre oxigênios, da estrutura secundária da montmorilonita, que lhe confere um caráter ácido de Brönsted. A acidez também é resultante da estrutura primária da argila, devido à presença de cátions metálicos $\left(\mathrm{Al}^{3+}, \mathrm{Mg}^{2+}\right)$, que atuam como sítios ácidos de Lewis. Além disso, outros cátions metálicos podem estar presentes na camada interlamelar da argila, agindo também como ácidos de Lewis.

A possibilidade de estabilizar intermediários de alta energia, de atuar como agente oxidante, redutor, bem como fotocatalíticos; e ainda, devido a sua estrutura lamelar aumentar a frequência de choques entre reagentes, na medida em que eles difundem-se na superfície da argila, são alguns aspectos que podem ser citados em relação à catálise promovida pela montmorilonita, K-10.

A superfície da argila com seus respectivos íons, forma uma dupla camada elétrica que é equivalente a um cátodo mergulhado em uma solução eletrolítica, faltando apenas a corrente elétrica contínua. Portanto, a reação de oxirredução, realmente, deve ocorrer nesta superfície, se a mesma contiver energia disponível, e essa energia tiver como fonte a reorganização do retículo cristalino da argila.

Obtenção de álcoois e formação de lactonas (ADAMS, 1987), aobtenção de compostos $\beta$-enamino carbonílicosa partir de $\beta$-dicarbonílicos(BRAIBANTE et al., 1994,1998 e 2003a); a alquilação e acilação de anéis aromáticos em presença de ácidos de Lewis (VARMA, 2002), e a obtenção de compostos heterocíclicos (BRAIBANTE et al., 2003b,1996, 1997 e 1999), são alguns exemplos de reações nas quais é utilizada a montmorilonita, K-10.

Sistemas resultantes da impregnação de montmorilonita, K-10, com nitrato de ferro III (Clayfen) e nitrato de cobre II (Claycop) são empregados em reações de oxidação com sucesso.

\section{O uso de K10 em reações Orgânicas}

A montmorilonita K10conhecida por apresentar acidez equivalente a alguns superácidos possui uma acidez de Hammet inferior a -8,2 quando tratada a 120 ${ }^{\circ} \mathrm{C}$ a baixa pressão. A acidez de Hammet baseia-se no seguinte equilíbrio:

$\mathrm{BH} \leftrightarrow \mathrm{B}+\mathrm{H}^{+}$e é dada por $\mathrm{H}_{0}=-\log \left(\mathrm{a}_{\mathrm{H}+} \cdot \mathrm{f}_{\mathrm{B}} / \mathrm{f}_{\mathrm{BH}}\right)$

Onde $\mathrm{a}_{\mathrm{H}+}$ é a atividade do íon $\mathrm{H}^{+}$em solução $\mathrm{e}_{\mathrm{fB}} / \mathrm{f}_{\mathrm{BH}}$ é a proporção dos coeficientes de atividade da base B e a base conjugada $\mathrm{BH}^{+}$. Sua acidez está relacionada com a eletronegatividade ou entalpia de hidratação dos cátions trocáveis (POLLET, 1983).

Uma das primeiras aplicações da montmorilonita como catalisador foino craqueamento de hidrocarbonetos na produção de gasolina.

A reação de alquilação do benzeno com cloreto de benzila fornece $72 \%$ de rendimento de difenilmetano em 4 horas de reação. Laszlo (LASZLO e MATHY, 1987) usando troca catiônica obteve $100 \%$ de conversão resultando $60 \%$ do produto monoalquilado e $30 \%$ do produto dialquilado. Ao empregar K-10 como suporte esta reação produziu $98 \%$ de conversão do cloreto de benzila após 15 minutos rendendo 50\% do difenilmetano. Este trabalho evidencia a grande influência do suporte na catálise, uma vez que os ácidos de Lewis apresentavam diferenças de atividades quando livres comparadas a quando suportados, estes resultados permitiram propor como ordem de reatividade a seguinte sequência para os ácidos de Lewis suportados em K-10:

$\mathrm{Fe}^{3+}>\mathrm{Zn}^{2+}>\mathrm{Co}^{2+}>\mathrm{Cr}^{3+}>\mathrm{Cu}^{2+}>\mathrm{Al}^{3+}>\mathrm{Ti}^{4+}$

Sendo esta ordem praticamente oposta à obtida para os ácidos livres.

A Montmorilonita, K10, atualmente vem sendo utilizada como catalisador em reações orgânicas em processos nos quais são utilizados ácidos de Lewis ou de Brönsted. (HOLMQUIST, et al., 1989). Como exemplo temos reações de esterificação de ácidos carboxílicos, a conversão de cetonas a enaminas, a condensação de anilina com $\beta$-ceto-ésteres, as reações de Diels-Alder, a oxidação de álcoois eas reações de condensação entre outras.

Obtenção de álcoois (ADAMS et al., 1982a) formação de lactonas (ADAMSet al.,1982b) obtenção de compostos $\beta$-enamino carbonílicos (BRAIBANTE et al., 2003b, 1996, 1997, 1999), a partir de $\beta$-dicarbonílicos; a alquilação e Acilação de anéis aromáticos em presença de ácidos de Lewis (VARMA, 2002) obtenção de compostos heterocíclicos (BRAIBANTE et al. 1996 e 1999), são alguns exemplos de reações onde a montmorilonita, K-10 é utilizada como suporte sólido.

Sistemas resultantes da impregnação de montmorilonita, K-10, com nitrato de ferro III (Clayfen) (BRAIBANTE et al 2007), e nitrato de cobre II (Claycop) (CORNELIS e LASZLO, 1986) são empregados em reações de oxidação com sucesso, associada ou não a irradiação de micro-ondas

\subsection{K10 na obtenção de enamino compostos}

O método clássico para a obtenção de compostos $\beta$-enamino carbonílicos, envolve a reação de condensação de compostos 1,3-dicarbonílicos com aminas primárias ou secundárias, em solventes aromáticos com remoção azeotrópica da água. Também pode ser utilizada amônia gasosa com $\beta$-dicarbonílicos em solução (OSTERCAMP, 1970). 
Quando são empregadas aminas pouco reativas procedimentos drásticos, como o uso de autoclaves e altas temperaturas, são utilizados. O inconveniente desses procedimentos é a alta proporção de subprodutos formados.

Devido à grande versatilidade destes compostos, vários estudos vêm sendo desenvolvidos na tentativa de obtê-los com grande pureza e bons rendimentos.

Braibante et al. (1994) elegendo a metodologia de reações em suporte sólido como método seletivo, versátil e simples, sintetizou $\beta$-enamino cetonas e ésteres acíclicos, empregando como suporte sólido montmorilonita, K-10 (Esquema 1).<smiles>[R]C(=O)CC(C)=O</smiles>

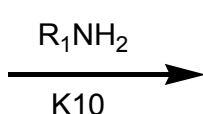<smiles>[R]N/C(C)=C\C([R])=O</smiles>

$\mathrm{R}=\mathrm{Me}, \mathrm{OEt}$

$\mathrm{R}_{1}=\mathrm{H}, \mathrm{Me}, \mathrm{Ph}, \mathrm{Bn}, i-\mathrm{Pr}, t-\mathrm{Bu}$

\section{Esquema 1}

Uma outra metodologia empregada para a obtenção de $\beta$-enamino cetonas foi utilização de suporte sólido - montmorilonita (K-10) -associado a metodologia de sonicação (ultrassom)(BRAIBANTE et al., 1998). O sistema 1,3-dicarbonílico usado foi ap-fenil substituídas e sistemas 1,3-dicarbonílicos derivados de 2-acilcicloalcanonas (Esquema 2).

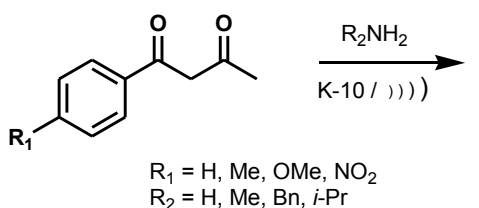<smiles>[R]c1ccc(C(=O)/C=C(/C)N([R2])[2H])cc1</smiles>

Esquema 2

Quando os sistemas 1,3-dicarbonílicos, derivados de 2-acilcicloalcanonas de 5 e 6 membros, foram utilizados nas reações de condensação em K10,(BRAIBANTE et al.,1998) foi observada uma regiosseletividade no ataque da amina ao centro eletrofílico. Assim, são obtidas, com a utilização de anéis de 5 membros enamino cetonas exocíclicas e, com anéis de 6 membros, enamino cetonas endocíclicas (Esquema 3).<smiles>[R]C(=O)C1CCCC1=O</smiles>

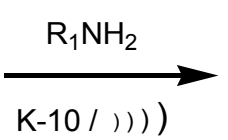

$20 \mathrm{~h}$<smiles>[R]C(=O)C1CCCCC1=O</smiles>

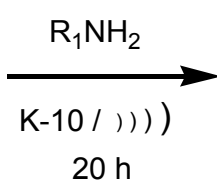<smiles>[R]NC1=C(C([R])=O)CCCC1</smiles>

$\mathrm{R}=\mathrm{Me}, \mathrm{Ph}$ $\mathrm{R}_{1}=\mathrm{H}, \mathrm{Me}, \mathrm{Bn}$

Esquema 3

Braibante et al (2003b), descreveram a síntese de compostos $\beta$-enamino carbonílicos através da condensação de um $\beta$-oxotioxoester com aminas primárias utilizando a metodologia de suporte sólido associada a ultrassom (Esquema 4).

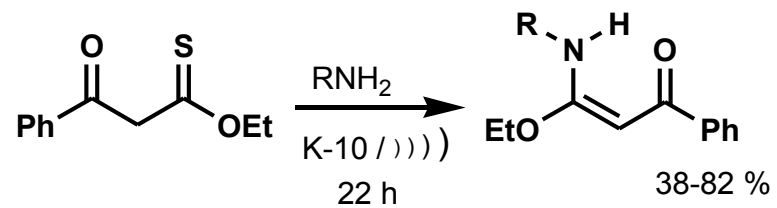

$\mathrm{R}=\mathrm{H}, \mathrm{Me}, \mathrm{Ph}, \mathrm{Bn}, i-\mathrm{Pr}$, Alil, $\mathrm{CH}\left(\mathrm{CH}_{3}\right) \mathrm{Ph}$

Esquema 4

A metodologia de obtenção de enamino compostos empregando o sistema K-10/S-Prolina, denominado em nosso laboratório de "ClayPro", (BRAIBANTE et al., 2012) apresenta bons rendimentos, regiocontrole e geometria $\mathrm{Z}$ dos enamino compostos, mantendo as vantagens do work-up das reações com suporte sólido, tais como redução do tempo de reação, condições reacionais brandas, o uso de solvente apenas no isolamento o que simplifica o isolamento a uma simples operação de filtração (Química verde) (Esquema 5). Esta metodologia usando catalisador bifuncional adsorvido em suporte sólido abre a perspectiva do emprego deste sistema em reações com indução assimétrica e/ou reações multicomponentes (MCR's).<smiles>[R]C(=O)C([R18])C(C)=O</smiles>

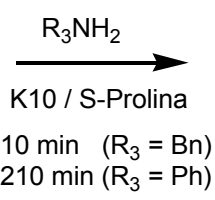<smiles>[R]C(=O)/C([R3])=C(/C)N([R9])[2H]</smiles>

II a-f

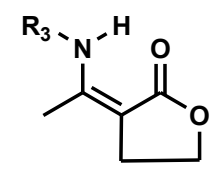

II g,h

\begin{tabular}{|l|l|l|l|c|}
\hline & $R_{1}$ & $R_{2}$ & $R_{3}$ & Rdto \% \\
\hline a & $H$ & $M e$ & $B n$ & 80 \\
\hline b & $H$ & $M e$ & $P h$ & 83 \\
\hline $\mathrm{c}$ & $M e$ & $M e$ & $B n$ & 86 \\
\hline d & $M e$ & $M e$ & $P h$ & 80 \\
\hline
\end{tabular}




\begin{tabular}{|l|l|l|l|l|}
\hline e & $H$ & OEt & $B n$ & 80 \\
\hline $\mathrm{f}$ & $\mathrm{H}$ & OEt & $\mathrm{Ph}$ & 90 \\
\hline $\mathrm{g}$ & \multicolumn{2}{|l|}{$-\mathrm{CH}_{2} \mathrm{CH}_{2} \mathrm{O}-$} & $\mathrm{Bn}$ & 95 \\
\hline $\mathrm{h}$ & \multicolumn{2}{|l|}{$-\mathrm{CH}_{2} \mathrm{CH}_{2} \mathrm{O}-$} & $\mathrm{Ph}$ & 76 \\
\hline
\end{tabular}

Esquema 5

\subsection{K10 - Reatividade de $\beta$-EnaminoCarboníli- $\cos$}

Compostos $\beta$-enamino carbonílicos são intermediários sintéticos muito versáteis, pois são pequenas moléculas polifuncionalizadas que apresentam dois centros eletrofílicos e três centros nucleofílicos. Sua reatividade pode ser avaliada frente a eletrófilos e nucleófilos, ou seja, através de reações de acilação, halogenação, redução e ciclização.

Braibante et al., (1994, 1997 e 1998), explorando a metodologia de reações sob suporte sólido, montmorilonita $\mathrm{K}-10$, utilizaram isocianato de fenila e isotiocianato de fenila como agentes acilantes de enamino compostos, e obtiveram somente produtos C-acilados (Esquema 6).
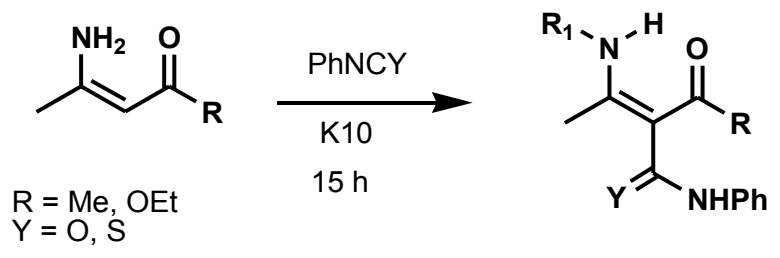

\section{Esquema 6}

A bromação de $\beta$-enamino compostos acíclicos e $\beta$-enamino cetonas cíclicas derivadas da dimedona, (BRAIBANTEet al1998) foi efetuada utilizando N-bromosuccinimida (NBS), montmorilonita-K10 e metanol, obtendo $\beta$-enamino compostos $\beta$-bromados (Esquema 7).<smiles>CC1(C)CC(=O)C=C(N)C1C(O)C1(C)CC(=O)C(Br)=C(N)C1</smiles>

$\mathrm{R}=\mathrm{H}, \mathrm{Me}, \mathrm{Ph}, \mathrm{Bn}, \mathrm{i}-\mathrm{Pr}, \mathrm{CH}\left(\mathrm{CH}_{3}\right) \mathrm{Ph}$, Alil<smiles>[R]C(=O)C=C(C)N</smiles>

$\mathrm{R}=\mathrm{Me}, \mathrm{OEt}$

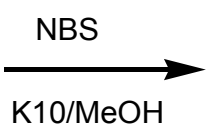

Esquema 7<smiles>[R]C(=O)/C(Br)=C(/C)N</smiles>

Braibante et al., $(1997,1999)$ sintetizaram pirazóis, isoxazóis, N-metil pirazolonas e isoxazolinonas a partir da ciclização de $\beta$-enamino compostos $p$-fenil substituídos com hidrazina, metil hidrazina e cloridrato de hidroxilamina, através de reações em meio homogêneo, refluxo em etanol, comparando com a metodologia de reações sob suporte sólido, montmorilonita, K-10, associada ou não a ultrassom. Evidenciando a influência do catalisador (Esquema 8 e 9).

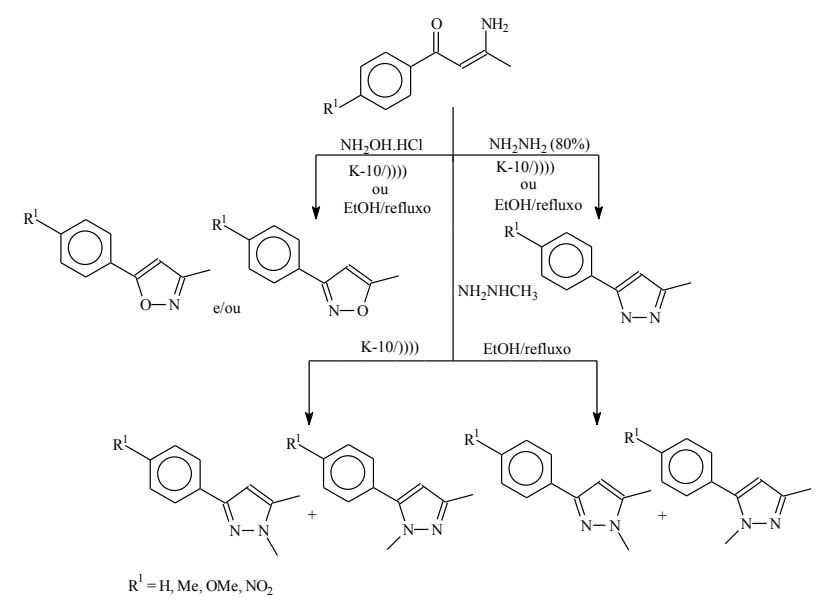

Esquema 8

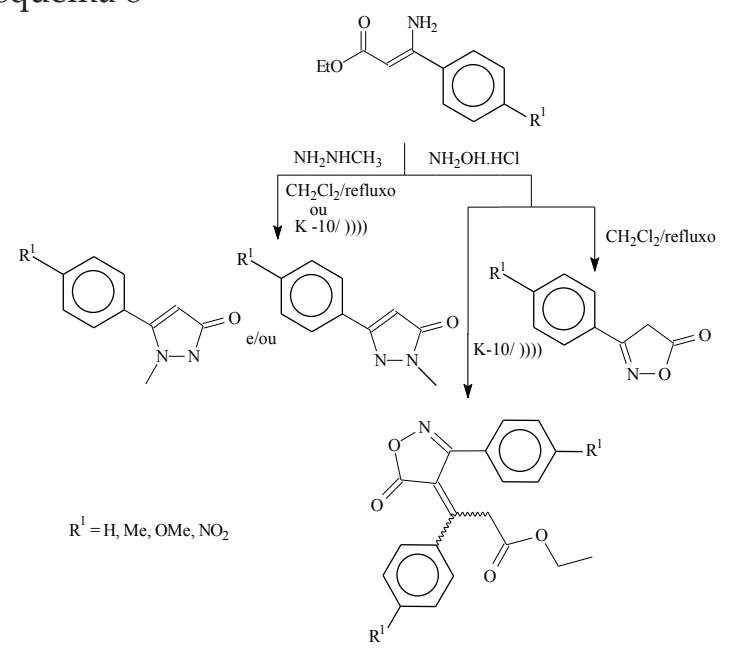

Esquema 9

Na obtenção de aminopirazóis,Braibante et al., (2003) utilizaram uma série de $\alpha$-oxocetenos $\mathrm{O}, \mathrm{N}$ substituídos para reagir com hidrazina, utilizando a metodologia de suporte sólido, montmorilonita, K-10, associada a ultrassom (Esquema 10).

Esquema 10

\subsection{O uso de K10 -Micro-ondas}

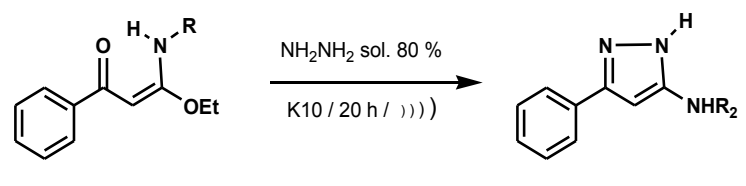

$\mathrm{R}=\mathrm{H}, \mathrm{Me}, \mathrm{Ph}, \mathrm{Bn}, \mathrm{i}-\mathrm{Pr}, \mathrm{CH}\left(\mathrm{CH}_{3}\right) \mathrm{Ph}$, Alil

O primeiro trabalho de grupo brasileiro aplicando 
a irradiação de micro-ondas associado a catálise em suporte sólido, na área de Química Orgânica, data de 2003e foi publicado no Journal Brazilian Chemical Society. O trabalho relata os resultados obtidos para preparação de $\beta$-enaminonas (BRAIBANTE et al., 2003a) a partir de compostos dicarbonílicos pela utilização da argila K-10 sob irradiação de micro-ondas (forno doméstico SANYO EM-700T, $700 \mathrm{~W}$ ) com tempos de reação que variaram de 2 a 7 minutos e rendimentos na faixa de 70 a $90 \%$.

Para a utilização de aminas de baixo ponto de ebulição, Braibante et al. (2003a) empregaram estas aminas sob forma de seus acetatos de amônio derivados (Esquema 11).<smiles>[R7]C(=O)CC(C)=O</smiles><smiles>[R]C(=O)/C(Cl)=C(/C)NC(=O)C(Cl)C(=O)ON</smiles><smiles>CC1(OC(=O)C2C(=O)CC(=O)CC2(C)C)CC(=O)C=C(N)C1</smiles>

$\mathrm{R}=\mathrm{H}, \mathrm{Me}, \mathrm{Ph}, \mathrm{Bn}, \mathrm{i}-\mathrm{Pr}, \mathrm{CH}\left(\mathrm{CH}_{3}\right) \mathrm{Ph}$, Alil

\section{Esquema 11}

Em algumas rotas sintéticas para sistematizar a reatividade do grupo carboxílico, a reação de transesterificação ganha importância quando podemos associar grupos que induzam regiosseletividade.

Duas metodologias podem ser citadas para efetuar a transesterificação do composto 3a Esquema 12, frente aos álcoois alílico, benzílico e $(1 S, 2 R, 5 S)-(+)$-mentol. Uma delasconsiste em adicionar quantidades equivalentes (3 $\mathrm{mmol}$ ) do $\beta$-cetoéster 3a e do álcool em questão, juntamente com tolueno previamente destilado e montmorilonita (K-10), em quantidades catalíticas (100 mg/mmol)(JIN et al., 2002). A mistura reacional foi deixada sob refluxo durante $12 \mathrm{~h}$, eretirado o solvente usando evaporador rotativo.Em um estudo comparativo (BRAIBANTE et al., 2003) empregou a metodologia de K-10 associado a micro-ondas que consiste em adicionar quantidades equivalentes ( $3 \mathrm{mmol}$ ) do $\beta$-cetoéster 3a e do álcool em questão, juntamente com $300 \mathrm{mg}$ de montmorilonita (K-10), em frasco de $10 \mathrm{~mL}$, e submeter à irradiação de energia de micro-ondas, em forno doméstico, na potência de 650 watts (Esquema 12). O tempo reacional variou de 15 a 18 minutos.

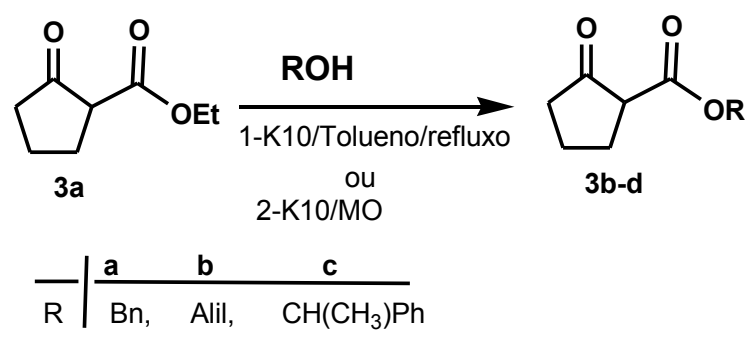

Esquema 12

A oxidação de álcoois secundários, utilizando $\mathrm{KMnO}_{4}$ adsorvido em $\mathrm{CuSO}_{4}$ ou K-10 empregando irradiação de micro-ondas (MO) em reações sem solventes foi efetuada por Braibante etal. (2005). Esta metodologia foi empregada pelo grupo para a oxidação de $\alpha$-hidroxicetonas em estudo comparativo dos métodos de oxidação usando Clayfen (Nitrato de ferro adsorvido em K10) e Claycop (Nitrato de cobre adsorvido em K10) (BRAIBANTE etal., 2007), com significativa redução de tempo e melhores rendimentos quando empregado o Claycop (Tabela 1).

A aplicação de suportes sólidos, como a argila Montmorilonita (K10) na função de catalisadores para reações químicas constitui um componente essencial para a química verde.

Tabela 1 - Comparação entre as metodologias de oxidação

\begin{tabular}{|c|c|c|c|}
\hline $\begin{array}{l}\text { Tempo(m } \\
\text { Rend.\% }\end{array}$ & / Potenc. & atts) / & 12-dicetona \\
\hline $\mathrm{KMnO}_{4} / \mathrm{Cu}$ & Clayfen & Claycop & \\
\hline $15 / 500 / 55$ & $\begin{array}{l}3 / 600 \\
/ 50\end{array}$ & $3 / 500 / 50$ & \\
\hline $18 / 500 / 55$ & $\begin{array}{l}10 / 300 / 8 \\
1\end{array}$ & $3 / 500 / 90$ & \\
\hline $15 / 500 / 50$ & $\begin{array}{l}10 / 300 / 6 \\
0\end{array}$ & $3 / 500 / 60$ & \\
\hline $15 / 500 / 60$ & $\begin{array}{l}10 / 300 / 6 \\
0\end{array}$ & $3 / 500 / 65$ & \\
\hline
\end{tabular}

A aplicação de suportes sólidos, como a argila Montmorilonita (K10) na função de catalisadores para reações químicas constitui um componente essencial para 
a química verde. O uso da irradiação de micro-ondas para promover reações químicas mais rápidas e limpas é também um importante componente da química verde, especialmente quando usado em combinação com argilas e condições livres de solventes (LASZLO 1987). Um exemplo simples de reação on-pot, isenta de solventes é a síntese do composto metilenodioxiprecoceno (MDP), um inseticida natural com atividade de hormônio anti-juvenil em alguns insetos (Antijuvenóides, antagonistas do hormônio juvenil conhecido como precocenos). A síntese consiste em uma condensação de sesamol (encontrado no óleo de gergelim) com 3-metil-2-butenal catalisada por argila K-10, assistida por micro-ondas (MATTHEW et al., 2006) (Esquema 13). Este é um procedimento apropriado para a incorporação no currículo de graduação em química orgânica experimental pois constitui um exemplo peculiar de química verde em ação.

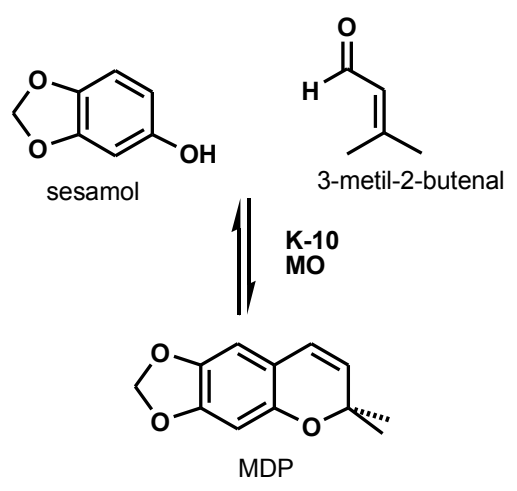

Esquema 13

\section{Considerações Finais}

A Montmorilonita, K10, é utilizada como catalisador em diferentes tipos de reações orgânicas. As reações que necessitam catalisadores como ácidos de Lewis ou ácido de Brönsted, podem ser efetuadas em alta eficiência usando K-10. Entre as aplicações podemos destacar o uso como suporte sólido para ancorar reagentes, comoos oxidantes Clayfen e Claycop, ou impregnado de metais como $\mathrm{Fe}^{3+}, \mathrm{Zn}^{2+}, \mathrm{Co}^{2+}$, usados em reações de condensação, ou MCR's e atualmente ancorando a prolina e atuando na função de catalisador bifuncional. Entre tantas diversidades foi enfocada a possibilidade de usar as reações em K10 associadas a várias formas de energia, refluxo em solventes orgânicos, em micro-ondas ou ultrassom e atendendo a preceitos da química verde em reações sem solventes. Várias rotas sintéticas e interconversão de grupos funcionais foramdestacadas como a transesterificação, halogenação, oxidação aliadas as clássicas reações de acilação e alquilação de compostos aromáticos, reação de Diels Alder e rearranjos que levam a sistemas heterocíclicos como Pirazóis, Isoxazóis, Isoquinolinas, Pirazolonas e Isoxazolonas foco de nosso grupo. Todas estas propriedades e aplicações ressaltam a versatilidade do K-10, como suporte sólido, em reações orgânicas e sua influência na quimio e regiosseletividade.

Outro aspecto deste trabalho foi a possibilidade de ressaltar o fato que em 1989 nosso grupo enviou a revista Química Nova, (publicado em 1990), o primeiro artigodo grupo enfocando suporte sólido, concebido integralmente na infraestrutura do CCNE-UFSM (Braibante, et.al., 1990). Agora na comemoração dos 25 anos de contribuição do grupo nesta área, estamos marcando nossa atuação com este artigo na revista Ciência \& Natura, nos seus 25 anos, uma feliz coincidência em nossas caminhadas.

\section{Referências}

ADAMS, J.M.; GRAHAM, S.H.; CLEMENT, D.E., (1982a) Reactions of alcohols with alkenes Clays and Clay Minerals, 30, 129, 1982

ADAMS, J.M.; Davies, M.; Graham, S.H.; Thomas, J.M., (1982b).; J. Catalysis, 78, 179, 1982

BRAIBANTE, M.F.; BRAIBANTE, H.S.; SALVATORE, S.J.S.A, Síntese de enamino compostos utilizando suportes sólidos, Química Nova, 13,67,1990

BRAIBANTE, M.E.F.; BRAIBANTE, H.T.S.; MISSIO, L., ANDRICOPULO, A.; Synthesis and reactivity of $\beta$-amino unsaturated ketones and esters using K10; Synthesis, 898.1994

BRAIBANTE, M.E.F.; BRAIBANTE, H.T.S.; VALDUGA, C.; SQUIZANI, A, Synthesis, 1019,1998

BRAIBANTE, M.E.F.; BRAIBANTE, H.T.S.; ROSSO, G.B.; ORIQUES, D.A., (2003a), Preparation of $\beta$-Enamino Carbonylic Compounds using Microwave Radiation/K-10. J. Braz. Chem. Soc., 6, 994, 2003

BRAIBANTE, M.E.F.; BRAIBANTE, H.T.S.; ROZA, J.K.; HENRIQUES, D.M.; TAVARES, L.C., (2003b), Synthesis, 8, 1160, 2003

BRAIBANTE, M.E.F.; BRAIBANTE, H.T.S.; ROSSO, G.B.; ROZA, J.K., SYNTHESIS, 13, 1935. 2001

BRAIBANTE, M.E.F.; BRAIBANTE, H.T.S.; MISSIO, L., J. Heterocyclic Chem., 33, 1243. 1996

BRAIBANTE, M.E.F.; BRAIBANTE, H.T.S.; VALDUGA, C., Reactivity of p-Phenil Substituted Enamino compound using K10/ultrasound I. Synthesis of Pyrazoles and Pyrazolones J. Heterocyclic Chem., 34, 1453, 1997

BRAIBANTE, M.E.F.; BRAIBANTE, H.T.S.; 
SANTIS, D.; VALDUGA, C., Reactivity of p-Phenil Substituted Enamino compound using K10/ultrasound II. Synthesis of Isoxazol and Isoxazolones, J. Heterocyclic Chem. 36, 505, 1999

BRAIBANTE H.T.S; BRAIBANTE, M.E.F.; COSTA C.C.; WOLENHAUPT, S; Oxidação de alfahidroxicetonas usando reagentes em K10/MO,30 a Reunião Anual da Sociedade Brasileira de Química $-2007$

BRAIBANTE, M.E.F.; BRAIBANTE, H.T.S.; COSTA, C. C.; MARTINS, D.B., Reactivity of chloroacetylated $\beta$-enamino compounds. Tetrahedron Lett., 43, 8079. 2002

BRAIBANTE, M.E.F.; BRAIBANTE, H.T.S.; ROSSO, GMONTEIRO, F., Synthesis and $\alpha$-Bromination of b-enamino Compounds using K10. 8th BMOS -São Pedro, SP, 1998

BRAIBANTE H.T.S; BRAIBANTE, M.E.F.; ULIANA M.F.; PORTE L.F.-28aㅡ Reunião Anual daSociedade Brasileira de Química. 2005

BRAIBANTE H.T.S; BRAIBANTE, M.E.F.; COSTA C.C.; WOLENHAUPT, S; 30aํㄹ Reunião Anual da Sociedade Brasileira de Química - 2007

BRAIBANTE H.T.S; BRAIBANTE, M.E.F., SOSSMEIER T., FRIZZO, M., CARMO, R.; Obtenção de Enamino Compostos usando o sistema K-10/Prolina, XIX Encontro de Química da Região Sul Tubarão, SC- 2012

CORNELIS, A.; LASZLO, P., Preparative Organic Chemistry Using Clays, in Chemical Reactions in Organic and Inorganic Constrained Systems, 213, 1986.

HOLMQUIST, C.R.; ROSKAMP, E.J., A selective method for the direct conversion of aldehydes into keto-esters, J. Org. Chem., 54, 3258, 1989

JIN, T.; ZHANG, S.; LI, T., Transesterification of $\beta$-ketoesters with alcohols catalyzed by montmorillonite K-10, Green Chem., 4, 32, 2002

LASZLO P.; Chemical reactions on clays, Science, 235, 1473, 1987

LINDSTRÖM, P.; TIERNEY, J.; WATHEY, B.; WESTMAN, J.; Microwave Assisted Organic Synthesis: A Review Tetrahedron, 57, 9225, 2001

MATTHEW R. DINTZNER, PAUL R. WUCKA, AND THOMAS W. LYONS, Microwave-Assisted
Synthesis of a Natural Insecticide on Basic Montmorillonite K10 Clay.; J. Chem. Educ, 270, 2006

MERRIFIELD, R.B, Solid Phase Peptide Synthesis. I. The Synthesis of a Tetrapeptide. J. Am. Chem. Soc., 1963, 85, 2149. 1963

OSTRCAMP, D. Vinylogous imides II, J. Org. Chem.35, 1632, 1970.

POLLET, P.L.,3-Ketoesters by malonic synthesis. J. Chem. Educ., 60, 244. 1983.

POSNER, G.H. Organic Reactions at Alumina Surfaces; Angew. Chem. Int. Ed. Engl. 17, 487, 1978

PAULING, L., The structure of the chlorites, Proc. Natl. Acad. Sci., 36, 578, 1930

VARMA, R.S.; Clay and Clay supported reagents in Organic synthesis, Tetrahedron, 58, 1235, 2002. 